\title{
Hair cortisol as a novel biomarker of HPA suppression by inhaled corticosteroids in children
}

\author{
Laura Smy ${ }^{1,2}$, Kaitlyn Shaw ${ }^{3-5}$, Anne Smith ${ }^{3-5}$, Evan Russell ${ }^{6}$, Stan Van Uum ${ }^{6}$, Michael Rieder ${ }^{6}$, Bruce Carleton ${ }^{3-5}$ and \\ Gideon Koren ${ }^{1,2,6}$
}

\begin{abstract}
BACKGROUND: Asthma is the most common chronic condition in childhood, and the recommended pharmacotherapy for long-term control includes the use of inhaled corticosteroids (ICS). ICS were designed to act at the site of inflammation in the lung, thus decreasing systemic absorption and reducing the risk of adverse effects associated with corticosteroid use (e.g., HPA suppression and its consequent effects). Available data show that measurement of hair cortisol successfully reflects endogenous cortisol levels. We sought to examine whether hair cortisol measurements can be used to identify HPA suppression surrounding ICS therapy in children with asthma.
\end{abstract}

METHODS: Hair samples were collected from the vertex posterior region of the head of 18 asthmatic children. We compared their hair cortisol concentration during ICS use with the concentration prior to ICS use.

RESULTS: During ICS therapy, median hair cortisol levels were twofold lower compared with the period of no ICS use (median $89.8 \mathrm{ng} / \mathrm{g}$ vs. $198.2 \mathrm{ng} / \mathrm{g}, P=0.0015)$.

CONCLUSION: Hair cortisol is an effective biomarker of the HPA suppression associated with ICS therapy and can be a sensitive tool for determining systemic effects of ICS use and monitoring adherence. Future research is needed to characterize the effect of untreated asthma on hair cortisol concentrations, if any.

A sthma is the most common chronic disease in childhood, affecting $\sim 10 \%$ of children between the ages of 2 and 7 y (1). Inhaled corticosteroids (ICS) are first-line antiinflammatory drugs used in the long-term treatment of asthma (2). Currently, there are five different generic ICS available in Canada: budesonide, fluticasone propionate, beclomethasone dipropionate, mometasone furoate, and ciclesonide. These agents are designed to achieve therapeutic levels through topical application at the receptor site in the lung, thereby providing a lower level of systemic absorption and decreased risk of adverse effects compared with systemic corticosteroid treatments (3). However, in children, there are still concerns that chronic use of these agents may lead to adverse effects such as stunted growth (4), or HPA suppression (5), which is the focus of our research. Previous studies have found an association between ICS use in children and decreased salivary cortisol (6), as well as incidents of adrenal crisis, a potentially life-threatening condition (7).

Presently, clinical laboratory measurements of systemic cortisol levels use blood, saliva, or urine samples, all of which reflect short-term periods of hours to days. Due to the circadian variation in cortisol levels, the use of these matrices requires multiple measurements to truly understand cortisol changes over time (8). Over the last decade, hair cortisol measurement has emerged as a promising biomarker of long-term changes in the HPA (9). Studies have shown that hair concentrations of cortisol parallel plasma levels. For example, in patients with Cushing syndrome, resection of the adenoma resulted in a steep decrease in plasma and urine cortisol levels, paralleled by a similar temporal decline in hair concentrations (10). Since hair grows at an average rate of $1 \mathrm{~cm}$ per month $(\mathrm{cm} / \mathrm{mo})$ at the vertex posterior region of the head (11), a segmental analysis of hair is able to reveal changes in cortisol levels over time (8). The aim of our pilot study was to examine whether hair cortisol can detect HPA suppression, specifically decreased cortisol release, after initiation of ICS use in children with asthma.

\section{RESULTS}

Hair samples from 18 children with asthma were analyzed. The patient demographics and description of ICS and concomitant steroid use are listed in Table 1. Further details for each individual patient regarding the type and duration of ICS use and the associated relative change in hair cortisol concentration are depicted in Figure 1. The median hair cortisol concentration was twofold lower during periods of ICS exposure compared with the control period with no exposure to ICS $(89.8 \mathrm{ng} / \mathrm{g}$ (IQR, 24.5-256.5) vs. 198.2 ng/g (IQR, 56.2-798.0); $P=0.0015$, Figure 2).

\footnotetext{
The last two authors contributed equally to this work.

${ }^{1}$ The Motherisk Program, Division of Clinical Pharmacology and Toxicology, The Hospital for Sick Children, Toronto, Ontario, Canada; ${ }^{2}$ Graduate Department of Pharmaceutical Science, Leslie Dan Faculty of Pharmacy, University of Toronto, Toronto, Ontario, Canada; ${ }^{3}$ Child \& Family Research Institute, Vancouver, British Columbia, Canada; ${ }^{4}$ Division of Translational Therapeutics, Department of Pediatrics, University of British Columbia, Vancouver, British Columbia, Canada; ${ }^{5}$ Pharmaceutical Outcomes Programme, BC Children's Hospital, Vancouver, British Columbia, Canada; ${ }^{6}$ Department of Physiology and Pharmacology, Western University, London, Ontario, Canada. Correspondence: Gideon Koren (gidiup_2000@yahoo.com)
} 
Table 1. Patient demographics $(n=18)$

\begin{tabular}{lc}
\hline Characteristic & Result \\
\hline Age (y), median (range) & $4.1(1.4-8.8)$ \\
Gender, F (M) & $15(3)$ \\
Duration of ICS use (mo), median (range) & $5.1(2.3-20.7)$ \\
ICS dose ${ }^{a}$, number of patients & 7 \\
Low & 9 \\
Low/moderate & 2 \\
Moderate & 1 \\
ICS frequency of use ${ }^{a}$, number of patients & 8 \\
Intermittent & 9 \\
Intermittent/continuous & \\
Continuous & 9 \\
Switched from one type of ICS to another during & \\
therapy, number of patients & \\
Types of ICS used, number of patients ${ }^{b}$ & 14 \\
Ciclesonide & 9 \\
Fluticasone propionate & 1 \\
Beclomethasone & $3(2 / 1)$ \\
Combination, total (mometasone/fluticasone &
\end{tabular}

Use of other corticosteroids during the periods assessed by hair cortisol results, number of patients

\begin{tabular}{|c|c|}
\hline Systemic ${ }^{c}$-total & 7 \\
\hline Prior to starting ICS & 2 \\
\hline After starting ICS & 6 \\
\hline$>1$ course & 1 \\
\hline Intranasal & 5 \\
\hline
\end{tabular}

\section{DISCUSSION}

Despite the perception that there is low systemic bioavailability of ICS compared with oral or intravenous steroids, there is evidence of unexpected ICS-induced adverse effects in children such as height deficits that persist into adulthood with long-term budesonide use (12). Moreover, children have been repeatedly documented as experiencing adrenal suppression, insufficiency, or crisis while receiving ICS $(6,7,13)$. These adverse effects depend on the dose, duration of therapy, and age at the initiation of therapy. Moreover, it is likely that the different ICS pharmacokinetic characteristics are a determinant of adverse effects given the impact of pharmacokinetic properties on systemic activity (4).

The measurements of hair cortisol have been shown to be a sensitive biomarker of Cushing syndrome $(10,14)$, as well as stress associated with chronic pain, myocardial infarction, congestive heat failure, and numerous other conditions (8). Changes in hair cortisol can be detected in hair within 2 mo of the corresponding changes in plasma cortisol. In the pediatric

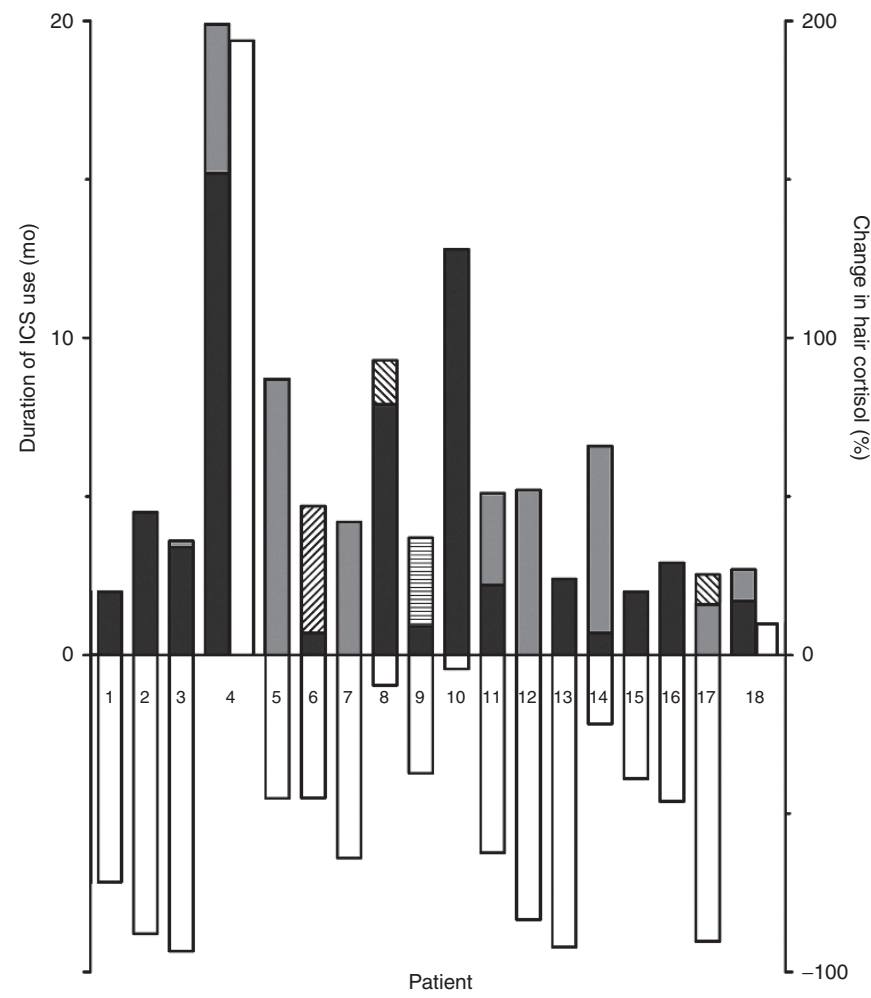

Figure 1. Individual patient inhaled corticosteroids (ICS) therapy duration and associated percent change in hair cortisol before ICS therapy compared to during ICS Therapy. Stacked bar graph displays the data for each of the 18 individual patients along the x-axis from left to right. Each patient's percent change in hair cortisol is indicated using the right $y$-axis and the white bar with the majority of children showing a negative change in hair cortisol. The duration of ICS use in months is indicated along the left $y$-axis with the bars extending in the positive direction. Each bar is coded to indicate the specific ICS used by the patient as follows: ciclesonide (black bar), fluticasone propionate (gray bar), beclomethasone dipropionate (left-downward hatched bar), mometasone furoate combination (right-downward hatched bar), and fluticasone propionate combination (horizontal hatched bar). Nine patients switched the type of ICS used, as shown by the change in color or pattern of the bar, and the hair segments that were analyzed for cortisol captured this switch. The bars for duration of ICS use also illustrate the length of the hair that was segmented for cortisol analysis during ICS therapy based on the average growth rate of $1 \mathrm{~cm} / \mathrm{mo}$, but it is an overestimation to a varying degree for each patient due to the exclusion of the hair segment when ICS was initiated.

context, this is a noninvasive method that makes it ethically more acceptable than drawing a blood sample by venipuncture. The ability of hair cortisol to serve as a sensitive biomarker of systemic suppression of the HPA may offer clinicians and researchers a time-sensitive tool to monitor the suppression of cortisol secretion in children with asthma who receive ICS, as well as in patients with other causes of decreased cortisol release.

Preliminary peer review reports suggested that ciclesonide may be associated with fewer adverse effects on growth in children (15), and adrenal suppression in adults $(16,17)$. Fewer observed adverse effects are believed to occur because ciclesonide is administered as a prodrug and activated by esterases in the lung, as well as its low oral bioavailability, rapid elimination, and high plasma protein binding (16). One article describing 


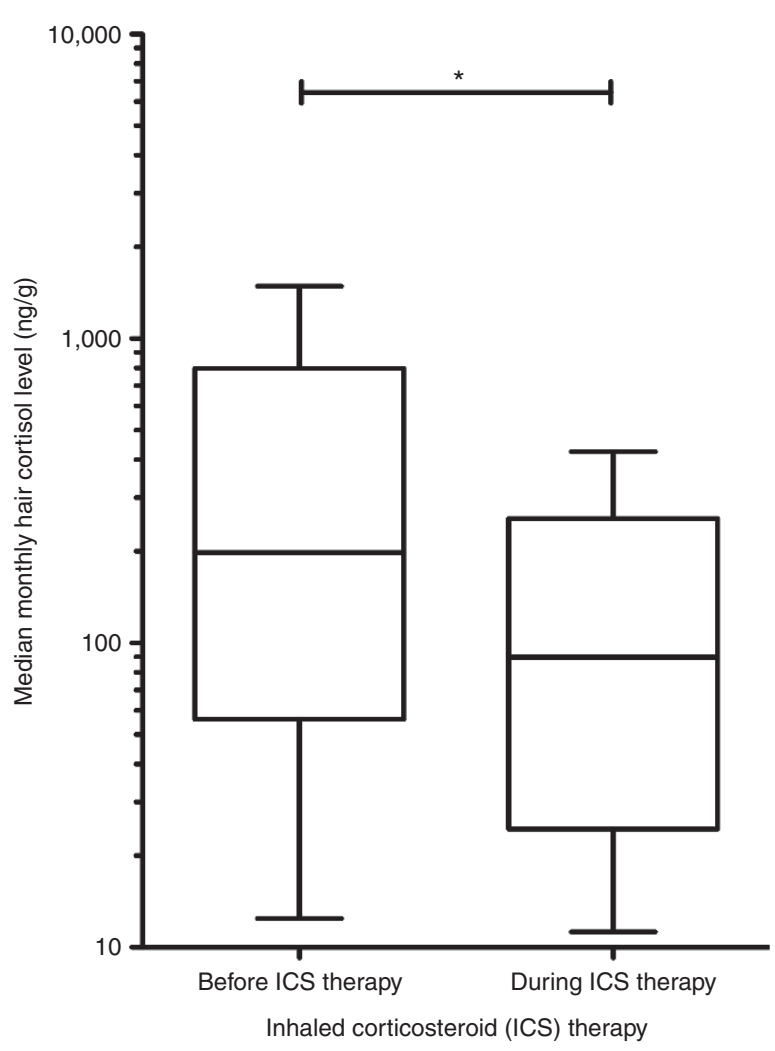

Figure 2. Comparison of median average monthly hair cortisol concentration before and during ICS therapy. Tukey boxplot comparison of the patients' median average monthly hair cortisol results for a maximum 6-mo period prior to ICS therapy and minimum 2-mo period during ICS therapy $(n=18) .{ }^{*} P=0.0015$.

four case studies with normalized adrenal cortisol release after switching from fluticasone to ciclesonide even suggests that ciclesonide use should be considered to potentially reverse adrenal suppression secondary to the use of other ICS (18). With 14 out of 18 children in our study using ciclesonide, 6 of whom have never used any other ICS, our findings contradict this sentiment and instead suggest that HPA suppression does occur with the use of ciclesonide.

The use of intranasal steroids and systemic steroids by some of our patients is a possible confounder. However, the use of intranasal corticosteroids has not been shown to decrease endogenous cortisol levels more than the use of ICS alone (6); and short-course systemic corticosteroid therapy for the treatment of asthma, as recommended by asthma treatment guidelines (19), was not associated with long-term suppression of adrenal function $(20,21)$. Furthermore, a hair cortisol analysis has the advantage of representing an overall picture of adrenal cortisol production and function of the HPA, rather than indicating acute or short-term fluctuations. Additional confounding may be present with the analysis of older hair segments. While the majority of studies have not found an association with the frequency of hair washing, some have reported declining hair cortisol in older hair segments (22). This may be in part due to repeated shampooing as found by Hamel et al. (23). Should this confounding be present in our study, our results would underestimate any decrease or overestimate any increase observed when comparing the hair cortisol levels prior to ICS use with those during ICS use.

The results of a recent study analyzing hair cortisol in children with asthma receiving ICS were similar to our findings with reported cortisol levels twofold lower than those in children without asthma (24). However, it was unknown whether the decrease in cortisol was due to the inflammatory condition itself or due to the effect of ICS. Our study addresses this issue by having the same children as the control group, which makes it less likely that the changes are due to the disease itself. As our study is a pilot investigation, it lacks the statistical power to discern dose- or product-dependent differences. Regardless, our results clearly show that hair cortisol is an effective biomarker of HPA suppression by ICS with respect to cortisol production.

In summary, hair cortisol is an effective biomarker of the HPA suppression associated with ICS therapy. Since hair grows $1 \mathrm{~cm} / \mathrm{mo}$ on average and although it takes new hair several days to surface, hair cortisol determination can serve as a sensitive tool for detecting adrenal suppression due to ICS therapy and for monitoring adherence, and potentially can be used for assessing the effects of changes in ICS type or dose. Additionally, it may be used in the investigation of other conditions associated with reduced cortisol release. Larger studies are needed to confirm and extend these findings. Furthermore, it will be important to compare hair cortisol of patients with asthma treated with ICS with that of disease-matched controls, as well as healthy controls, to fully discern the effect of the disease and the drug on hair cortisol levels.

\section{METHODS}

We identified children with asthma who had a history of at least 2 mo of ICS use preceded by a period of up to 6 mo without ICS, which could be captured by a hair sample. ICS use was categorized as intermittent if the ICS were prescribed for cold symptoms or seasonal use only, or continuous if prescribed for daily use. We did not exclude those who were exposed to short courses of systemic corticosteroids or intranasal corticosteroids. We categorized each patient's dose based on beclomethasone-hydrofluoroalkane equivalents as defined by Lougheed et al. (19). No patient in the study group was receiving high-dose ICS.

Full-length hair (40 or more strands) was collected noninvasively by cutting with scissors from the vertex posterior region of the head and sectioned into $2-\mathrm{cm}$ segments to capture the periods of exposure or no exposure to ICS, based on an average hair growth rate of $1 \mathrm{~cm} /$ mo. Hair cortisol was extracted and quantified by a validated enzymelinked immunosorbent assay method routinely used in our laboratory with a reported intraday and interday coefficient of variation of 3.8 and $8 \%$, respectively (25). A high correlation between the results of the enzyme-linked immunosorbent assay and those of liquid chromatography-mass spectrometry has been demonstrated $\left(r^{2}>0.9\right)(26)$, proving the validity of our cortisol measurement method.

For each participant, the average monthly hair cortisol for a maximum 6-mo period prior to ICS use and minimum 2-mo period during ICS use was determined. The hair segment corresponding to the period when ICS use was initiated was not included in the analysis to avoid variability in cortisol hair levels during the period of ICS buildup. The exclusion also aimed to reduce the influence of systemic corticosteroid exposure as this was frequently given at the time of starting ICS therapy. Because the average hair cortisol concentrations were not normally distributed, the median of the average monthly hair cortisol concentrations prior to and during the use of ICS was 


\section{Hair cortisol and HPA suppression}

compared using the Wilcoxon matched-pairs signed-rank test with a two-tailed $P$-value. The statistical analysis was performed using Prism software, version 5.0c (Graphpad Software, La Jolla, CA).

The research ethics boards of the University of British Columbia, Children's and Women's Health Centre of British Columbia, Western University, and The Hospital for Sick Children, Toronto, Ontario, Canada, approved the study. Written informed consent and assent were obtained from all participants and parents as appropriate and as specified by the respective research ethics boards.

\section{STATEMENT OF FINANCIAL SUPPORT}

This study was supported by The Canadian Institutes of Health Research Drug Safety and Effectiveness Network.

Disclosure: The authors have no conflicts of interest to declare.

\section{REFERENCES}

1. Thomas EM. Recent trends in upper respiratory infections, ear infections and asthma among young Canadian children. Health Rep 2010;21:47-52.

2. Hagan JB, Samant SA, Volcheck GW, et al. The risk of asthma exacerbation after reducing inhaled corticosteroids: a systematic review and metaanalysis of randomized controlled trials. Allergy 2014;69:510-6.

3. Weber B, Hochhaus G. A pharmacokinetic simulation tool for inhaled corticosteroids. AAPS J 2013;15:159-71.

4. Fuhlbrigge AL, Kelly HW. Inhaled corticosteroids in children: effects on bone mineral density and growth. Lancet Respir Med 2014;2:487-96.

5. Ahmet A, Kim H, Spier S. Adrenal suppression: A practical guide to the screening and management of this under-recognized complication of inhaled corticosteroid therapy. Allergy Asthma Clin Immunol 2011;7:13.

6. Heijsman SM, de Vries TW, Wolthuis A, Kamps AW. Salivary cortisol levels in prepubertal children using inhaled corticosteroids with or without concurrent intranasal corticosteroids. Pediatr Pulmonol 2011;46:1055-61.

7. Todd GR, Acerini CL, Ross-Russell R, Zahra S, Warner JT, McCance D. Survey of adrenal crisis associated with inhaled corticosteroids in the United Kingdom. Arch Dis Child 2002;87:457-61.

8. Russell E, Koren G, Rieder M, Van Uum S. Hair cortisol as a biological marker of chronic stress: current status, future directions and unanswered questions. Psychoneuroendocrinology 2012;37:589-601.

9. MeyerJS, NovakMA. Minireview: Hair cortisol:a novel biomarker of hypothalamic-pituitary-adrenocortical activity. Endocrinology 2012;153:4120-7.

10. Thomson S, Koren G, Fraser LA, Rieder M, Friedman TC, Van Uum SH Hair analysis provides a historical record of cortisol levels in Cushing's syndrome. Exp Clin Endocrinol Diabetes 2010;118:133-8.

11. Kronstrand R, Scott K. Drug incorporation into hair. In: Kintz P, ed. Analytical and Practical Aspects of Drug Testing in Hair. Boca Raton, FL: Taylor \& Francis Group, LLC, 2007:1-24.

12. Kelly HW, Sternberg AL, Lescher R, et al.; CAMP Research Group. Effect of inhaled glucocorticoidsin childhood on adultheight. NEnglJMed2012;367: 904-12.
13. Molimard M, Girodet PO, Pollet C, et al. Inhaled corticosteroids and adrenal insufficiency: prevalence and clinical presentation. Drug Saf 2008;31:769-74.

14. Manenschijn L, Koper JW, van den Akker EL, et al. A novel tool in the diagnosis and follow-up of (cyclic) Cushing's syndrome: measurement of long-term cortisol in scalp hair. J Clin Endocrinol Metab 2012;97: E1836-43.

15. Skoner DP, Maspero J, Banerji D; Ciclesonide Pediatric Growth Study Group. Assessment of the long-term safety of inhaled ciclesonide on growth in children with asthma. Pediatrics 2008;121:e1-14.

16. Takeda Canada Inc. Product Monograph - Alvesco(R) (ciclesonide inhalation aerosol). (http://www.takedacanada.com/ /media/Countries/ca/ Files/Product\%20PDFs/Alvesco\%20PM\%20ENG\%2017dec2012.pdf.). Accessed 7 June 2014.

17. Lipworth BJ, Kaliner MA, LaForce CF, et al. Effect of ciclesonide and fluticasone on hypothalamic-pituitary-adrenal axis function in adults with mild-to-moderate persistent asthma. Ann Allergy Asthma Immunol 2005;94:465-72.

18. Heller MK, Laks J, Kovesi TA, Ahmet A. Reversal of adrenal suppression with ciclesonide. J Asthma 2010;47:337-9.

19. Lougheed MD, Lemière C, Dell SD, et al.; Canadian Thoracic Society Asthma Committee. Canadian Thoracic Society Asthma Management Continuum-2010 Consensus Summary for children six years of age and over, and adults. Can Respir J 2010;17:15-24.

20. Ducharme FM, Chabot G, Polychronakos C, Glorieux F, Mazer B. Safety profile of frequent short courses of oral glucocorticoids in acute pediatric asthma: impact on bone metabolism, bone density, and adrenal function. Pediatrics 2003;111:376-83.

21. Jollin L, Thomasson R, Le Panse B, et al. Saliva DHEA and cortisol responses following short-term corticosteroid intake. Eur J Clin Invest 2010;40:183-6.

22. Wosu AC, Valdimarsdóttir U, Shields AE, Williams DR, Williams MA. Correlates of cortisol in human hair: implications for epidemiologic studies on health effects of chronic stress. Ann Epidemiol 2013;23:797811.e2.

23. Hamel AF, Meyer JS, Henchey E, Dettmer AM, Suomi SJ, Novak MA. Effects of shampoo and water washing on hair cortisol concentrations. Clin Chim Acta 2011;412:382-5

24. Kamps AW, Molenmaker M, Kemperman R, van der Veen BS, Bocca G, Veeger NJ. Children with asthma have significantly lower long-term cortisol levels in their scalp hair than healthy children. Acta Paediatr 2014;103:957-61.

25. Pereg D, Chan J, Russell E, et al. Cortisol and testosterone in hair as biological markers of systolic heart failure. Psychoneuroendocrinology 2013;38:2875-82.

26. Russell E, Kirschbaum C, Laudenslager ML, et al. Toward standardization of hair cortisol measurement: results of the first international interlaboratory round robin. Ther Drug Monit 2015;37:71-5. 